\title{
Efecto del pH sobre la proporción oocitaria de Paracheirodon axelrodi, en cautiverio.
}

The effect of $\mathrm{pH}$ on oocyte ratio for Paracheirodon axelrodi kept in captivity.

\section{Efeito do pH sobre a proporção oocitaria de Paracheirodon axelrodi, em cativeiro.}

\section{Estefany L. García-Cruz ; Karen T. Peña-Osorio ${ }^{1 *}$; Edwin Gómez-Ramírez ${ }^{2 *}$; Hernán Hurtado-Giraldo ${ }^{3 *}$}

BSc.

Esp, MSc.

$\mathrm{PhD}$

Grupo de Ictiología, Universidad Militar Nueva Granada, Facultad de Ciencias Básicas y Aplicadas, Bogotá, Colombia.

Email: estefanygc@gmail.com

\section{Resumen}

Paracheirodon axelrodi es la especie de pez ornamental más comercializado y exportado en Colombia. Los individuos comercializados son extraídos del ambiente afectando las poblaciones, lo que ha llevado a realizar estudios relacionados con su biología reproductiva para el desarrollo de protocolos de reproducción en cautiverio. En este estudio se evaluó el efecto de dos rangos de $\mathrm{pH}$ en el agua durante seis meses (T1: 5.5 - 5.8 y T2: 7.0 - 7.5), sobre las variables de crecimiento y la proporción de oocitos a nivel histológico en hembras adultas de $P$. axelrodi, para establecer si este parámetro afectaba el desarrollo gonadal y crecimiento a través del tiempo. De acuerdo a los resultados obtenidos, los rangos de $\mathrm{pH}$ no afectaron los porcentajes de los tipos de oocitos perinucleolar, alveolo cortical, vitelogénicos en etapa inicial y etapa tardía, ni en los oocitos en desarrollo de atresia; sin embargo, las condiciones mantenidas favorecieron las variables de crecimiento. Las proporciones oocitarias se mantuvieron similares durante el tiempo de experimentación. Los oocitos en vitelogénesis tardía son los que tuvieron la segunda mayor proporción celular desde el inicio hasta el final del experimento. Con este resultado se concluye que las condiciones a las que se sometieron los individuos de $P$. axelrodi, permitieron mantener hembras con un estado de madurez avanzado durante seis meses. Si se logra establecer el, o los parámetros desencadenantes de la maduración final y desove de las hembras, se tendrían individuos maduros por un periodo prolongado.

Palabras clave: ambiente, cautiverio, fisicoquímicos, histología, maduración, oocitos. 


\section{Abstract}

Paracheirodon axelrodi (cardinal tetra) is the most commercialised and exported ornamental fish species in Colombia. The fishes that are to be sold are taken from the environment there by affecting populations; this has led to studying their reproductive biology for developing protocols regarding reproduction in captivity. The present study evaluated the effect of two ranges of $\mathrm{pH}$ in water during a six-month period (T1: $5.5-5.8$ and T2: $7.0-7.5)$ regarding adult female $P$. axelrodi growth and oocyte rate at histological level for establishing whether this would affect gonad development and growth. The results showed that $\mathrm{pH}$ ranges did not affect the percentage of early and late perinucleolus stage, cortical-alveoli, vitelogenic oocytes, nor oocytes developing atresia; however, the conditions so maintained favoured variables regarding growth. Oocyte ratios were kept similar during experimentation time; late vitelogenesis oocytes had the second greatest cell ratio from the start to the end of the experiment. This result led to conclude that the conditions to which $P$. axelrodi were submitted allowed females to be kept in a state of advanced maturity for six months, and if the parameters triggering females' final maturation and spawning were established, then mature individuals could be had for a prolonged period.

Key words: environment, captivity, physicochemical, histology, maturation, oocyte.

\section{Resumo}

Paracheirodon axelrodi é a espécie de peixe ornamental mais comercializado e exportado na Colômbia. Os indivíduos comercializados são extraídos do ambiente afetando as populações, fato que levou a realizar estudos relacionados coma sua biologia reprodutiva para o desenvolvimento de protocolos de reprodução em cativeiro. No presente estudo avaliou-se o efeito de dois intervalos de pH da água (T1: 5.5 - 5.8 e T2: 7.0 - 7.5) durante seis meses, sobre as variáveis de crescimento e a proporção de oócitos a nível histológico em fêmeas adultas de $P$. axelrodi, para observar se este parâmetro afetava a maturação gonadal e o crescimento ao longo do tempo. De acordo com os resultados obtidos, os intervalos de $\mathrm{pH}$ não tiveram incidência nas porcentagens dos tipos de oócitos perinucleolar, alvéolo cortical, vitelogênicos na etapa inicial e etapa tardia nem nos oócitos em atresia, encontrados em ambos os tratamentos. No entanto, as variáveis foram favoráveis para o crescimento. As proporções oocitarias mantiveram-se similares durante o tempo experimental, sendo que os oócitos em vitelogênese tardiativeram a segunda maior proporção celular desde o inicio até o final do experimento. Com este resultado conclui-se que as condições que foram submetidosos indivíduos de $P$. axelrodi, permitiram manter fêmeas em um estado de maturação avançado durante seis meses, e assim como o pH, seria possível estabelecer outros parâmetros desencadeantes da maturação final e desova das fêmeas, mantendo assim indivíduos em maturação por um período prolongado.

Palavras chave: ambiente, cativeiro, físico-químicos, histologia, maturação, oócitos 


\section{Introducción}

Paracheirodon axelrodi, perteneciente a la familia Characidae, lidera la lista de peces ornamentales más comercializados por Colombia (SIPA, 2011). Se distribuye en los ríos de la Orinoquía colombiana, así como también en la zona media y alta del Río Negro en la Amazonía brasileña (Geisler y Annibal, 1987; Cooke, et al., 2009), en cuerpos de agua de baja profundidad, con corrientes lentas, poca incidencia Iumínica, conductividad eléctrica de 3 a $10 \mu \mathrm{S} / \mathrm{cm}$ y $\mathrm{pH}$ entre 4.5 - 5.0 (Ramírez et al., 2001).

$P$. axelrodi tiene gran acogida por los acuaristas, debido a su comportamiento en cardumen y su Ilamativa coloración: una franja horizontal de color azul y otra de color rojo intenso, desde el opérculo hasta el inicio de la aleta caudal (Ureña et al., 2007). Los ejemplares adultos de $P$. axelrodi alcanzan tallas entre los 3.1 a $3.5 \mathrm{~cm}$ de longitud total (Anjos y Anjos, 2006; Arias, et al; 2007) y el dimorfismo sexual no es evidente. Sin embargo, las hembras adultas suelen ser de mayor tamaño que los machos y su cuerpo presenta una forma más elíptica (Ureña, et al., 2007).

En Colombia, los ejemplares comercializados son extraídos del ambiente natural (Mancera y Álvarez, 2008), lo que podría causar un declive de las poblaciones de la especie. Lo anterior evidencia la necesidad de proponer trabajos de investigación dirigidos a conocer aspectos de su biología básica, relacionados con la reproducción y levante de ejemplares en cautividad, generando ambientes $\mathrm{y} / \mathrm{o}$ hábitats artificiales que favorezcan el desarrollo gonadal y eventual desove de la especie.

En los teleósteos, el crecimiento, la diferenciación y maduración gonadal están ligados principalmente a cambios fisicoquímicos y ambientales como el $\mathrm{pH}$, conductividad eléctrica, temperatura, fotoperiodo, entre otros. Estos cambios son percibidos por el pez a través de sistemas sensoriales (Sloman, 2011), que luego son transformados en impulsos nerviosos captados por dos zonas del cerebro implicadas en la reproducción: el hipotálamo y área pre óptica (Obando et al., 2013). Allí se produce el factor liberador de gonadotropinas $(\mathrm{GnRH})$, que a su vez tiene receptores en la hipófisis para la producción de hormonas FSH y $L H$, involucradas en el desarrollo, crecimiento y maduración gonadal (Lubzens, et al., 2010; Reading y Sullivan, 2011; Obando, et al., 2013).

En el medio natural para especies tropicales como $P$. axelrodi, son importantes las épocas de alta precipitación y las consiguientes inundaciones en las riveras, ya que alteran las condiciones fisicoquímicas del agua de los ríos. Es así, como trabajos realizados por Burton (1997), quien evaluó diferentes aspectos que influyen en el desove de $P$. axelrodi, muestran que la calidad del agua tiene un efecto sobre el evento reproductivo de la especie. Al implementar un $\mathrm{pH}$ de 5.5 y una conductividad eléctrica entre $40-60 \mu \mathrm{S} / \mathrm{cm}$, se logra el desove de los individuos, aunque con un número bajo de huevos fértiles. Así mismo, Anjos y Anjos (2006) reportan la inducción a desove bajo la combinación de las siguientes condiciones: adición de agua (simulando lluvia), temperatura de $26.0 \pm 1.0$ ${ }^{\circ} \mathrm{C}, \mathrm{pH}$ de $5.5 \pm 0.4$, conductividad eléctrica de $25 \pm 8 \mu \mathrm{S} / \mathrm{cm}$ y niveles de oxígeno disuelto alrededor de los $6.5 \mathrm{mg} / \mathrm{l}$. Los dos estudios reportan que los eventos de desove son esporádicos o con bajo número de huevos viables, y aún no es claro el efecto que cada variable fisicoquímica tiene sobre la maduración y posterior desove de la especie. Por esta razón, este estudio trató de establecer si existía un efecto por parte de la variable de $\mathrm{pH}$ sobre la proporción oocitaria en hembras adultas de $P$. axelrodi. Para ello, se evaluaron dos rangos de $\mathrm{pH}$ en el agua, determinando el resultado a través de la histología gonadal. 


\section{Materiales y métodos}

\section{Diseño experimental}

Este proyecto se realizó en el laboratorio de Embriología y Fisiología Animal de la Universidad Militar Nueva Granada, ubicada en el municipio de Cajicá (Cundinamarca Colombia). En el estudio se emplearon 160 ejemplares de $P$. axelrodi, que fueron adquiridos en una tienda autorizada para la venta de mascotas. Los individuos tenían una talla mínima de $2 \mathrm{~cm}$ de longitud total, que corresponde a individuos adultos según Anjos y Anjos (2006).

Para observar el efecto del $\mathrm{pH}$, se evaluaron dos tratamientos, con dos replicas, por un periodo de seis meses (T1: $\mathrm{pH}$ entre $5.5-5.8 \mathrm{y}$ T2: $\mathrm{pH}$ entre 7.0 - 7.5). Los rangos de $\mathrm{pH}$ establecidos obedecen a las variaciones que ocurren en este parámetro, desde la captura de los ejemplares en su hábitat natural $(\mathrm{pH}$ entre 5.5 - 5.8), transporte y finalmente acopio por parte de los acuaristas ( $\mathrm{pH}$ entre $7.0-7.5)$; factores que inciden en diferentes aspectos fisiológicos de los ejemplares (Mancera y Álvarez, 2008).

Inicialmente, los peces fueron aclimatados y mantenidos en cuarentena durante dos semanas en un acuario de $80 \mathrm{l}$. Posteriormente fueron distribuidos al azar en grupos de 40 individuos en acuarios de 40 I. Con el fin de disminuir la incidencia lumínica, cada acuario fue adecuado con un bio-filtro de caja, una capa de grava de aproximadamente $5 \mathrm{~cm}$ de altura y las paredes cubiertas. La temperatura se reguló con termostatos y la aireación se proporcionó por medio de una turbina GF 250 (280 Watt). Los peces fueron alimentados tres veces al día con alimento comercial de 45\% de proteína bruta al 5\% de la biomasa total.

\section{Condiciones fisicoquímicas del agua}

Cada acuario se mantuvo con algunas condiciones reportadas por Anjos y Anjos (2006) para el mantenimiento en cautiverio de $P$. axelrodi, nivel de oxígeno disuelto alrededor de $5.5-6.5 \mathrm{mg} / \mathrm{l}$ y temperatura entre 28 - $30^{\circ} \mathrm{C}$; no obstante, la conductividad eléctrica se mantuvo alrededor de 200 - 300 $\mu \mathrm{S} / \mathrm{cm}$. Debido al uso de agua declorada del sistema de acueducto municipal, esta variable no afecto la sobrevivencia de los peces.

Los productos nitrogenados fueron mantenidos en las concentraciones reportadas por Oliveira et al. (2008), como apropiadas para la sobrevivencia de la especie en cautiverio: nitrógeno amoniacal total (NAT), < $1.4 \mathrm{mg} / \mathrm{l} ; \mathrm{y}$ nitrito $\left(\mathrm{NO}_{2}^{-}\right)_{1}<0.3 \mathrm{mg} / \mathrm{l}$. Estos parámetros fueron medidos semanalmente con kit colorimétrico Tetra ${ }^{\circledR}$ y quincenalmente se realizó el $20 \%$ de recambio de agua del acuario. El pH se registró diariamente con un potenciómetro Schott Handy lab 2.

El agua de acueducto usada en el experimento registraba un $\mathrm{pH}$ inicial de alrededor 6.5 , por lo que para mantener el pH entre 5.5 - 5.8 (T1) se hizo un cambio gradual empleando $0.35 \mathrm{~g} / \mathrm{l}$ de fosfato de sodio monobásico $\left(\mathrm{NaH}_{2} \mathrm{PO}_{4} \cdot \mathrm{H}_{2} \mathrm{O}\right.$ Mallinckrodt), y para el $\mathrm{pH}$ entre 7.0 - 7.5 (T2) se utilizó bicarbonato de sodio y/o recambios de agua.

Los peces fueron mantenidos durante180 días, tiempo en el cual se registraron variables de crecimiento mensuales; los individuos fueron pesados en una balanza analítica (Ohaus Pioner) y se registraron medidas de longitud, total (LT) y estándar (LS), con un calibrador.

\section{Muestreo y procesamiento de muestras}

Teniendo en cuenta las guías para el manejo ético de peces para la investigación (AVMA, 2007), se sacrificaron, mensualmente, 10 individuos por tratamiento, escogidos de forma aleatoria. Los ejemplares fueron anestesiados con benzocaína $0.1 \mathrm{~g} / \mathrm{l}$ y 
sacrificados por corte medular a nivel cervical (Tovar, et al., 2009; Gómez et al., 2011).

Posterior al sacrificio, se les hizo un corte en la región abdominal y se fijaron en formaldehído al 4\%, amortiguado con PBS $(\mathrm{pH} 7.2$ - 7.4) durante ocho días a $4{ }^{\circ} \mathrm{C}$. Bajo el estereoscopio, se diseccionó a los ejemplares, observando el contenido visceral para identificar la presencia de la gónada a nivel macroscópico. Una vez extraídas las muestras gonadales, se sometieron a un proceso de deshidratación e inclusión en parafina (Tovar, et al., 2009; Gómez, et al., 2011).

Finalmente se realizaron cortes sagitales de 5 $\mu \mathrm{m}$ de espesor en un micrótomo (CUT SLEE 4060). Por individuo, se obtuvieron 10 láminas semiseriadas que abarcaron todo el tejido gonadal, cada una con 10 cortes. Entre lámina y lámina se descartaron un total de 450 micras. Los cortes fueron teñidos con la técnica de Mayer y Harris hematoxilina-eosina (HyE). Finalmente se realizó el montaje de los cortes en láminas con citorresina (Tovar, et al., 2009; Gómez, et al., 2011).

\section{Parámetros histológicos para la determinación de los estadios de madurez gonadal y proporciones oocitarias.}

La observación histológica de cada uno de los cortes se llevó a cabo a partir de los parámetros descritos para hembras según, Tyler y Sumpter (1996), Ravaglia y Maggese (2002), Brito y Bazzoli (2009) y Lubzens et al. (2010). En la observación del desarrollo oocitario se tuvieron en cuenta los cambios celulares ocurridos en el tamaño celular, el número de nucléolos, la acumulación de vitelo, la afinidad por HyE, la vacuolización del citoplasma, la presencia de alvéolos corticales, los cambios en las membranas oocitarias, la presencia de micrópilo, la formación de oocitos en estado atrésico y los folículos postovulatorios.
Una vez se identificaron los tipos celulares presentes en el tejido gonadal de las hembras, se realizó un conteo por tipo celular, teniendo en cuenta que los oocitos no se repitieran. El criterio usado para el conteo de oocitos fue la observación del núcleo y la membrana celular definida. Posteriormente, los datos obtenidos fueron transformados a porcentaje para establecer la proporción de cada tipo.

De acuerdo a las proporciones oocitarias encontradas, las hembras fueron clasificadas en 3 fases de madurez gonadal: previtelogénesis (oogonias y oocitos perinucleolar), vitelogénesis o en maduración (ooocitos alveolo cortical, y vitelogénicos) y hembras maduras (oocitos con núcleo migrado y formación de micrópilo).

\section{Análisis estadístico}

Los datos obtenidos por muestreo en cada tipo celular y variables de crecimiento (peso, longitud total y estándar), se sometieron a la prueba de normalidad Shapiro-Wilk. Una vez se determinó si presentaban un comportamiento normal y homogeneidad de varianzas, se realizó una prueba de t para establecer diferencias significativas entre tratamientos. De igual forma, se hizo una prueba de ANOVA de una vía, para determinar cambios en los porcentajes por tipo celular a través del tiempo y, en caso de existir diferencias, se realizó prueba Tukey.

Para las pruebas estadísticas se consideró el valor de $\mathrm{P}<0.05$ como estadísticamente significativo. El análisis estadístico se realizó con el programa $R$, versión 2.14.0, de libre distribución en internet (http://rproject.softonic.com/). 


\section{Resultados}

Descripción histológica
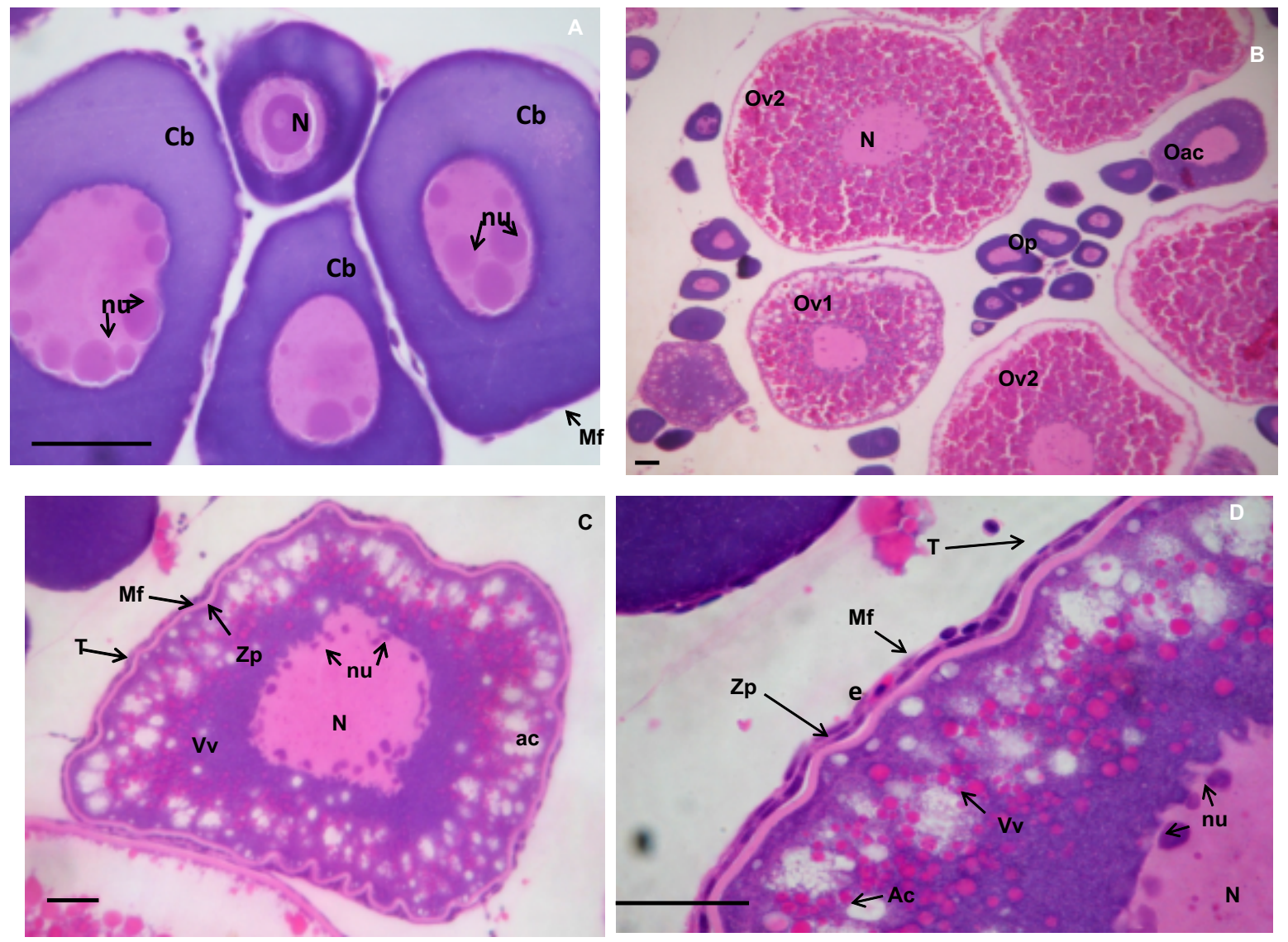

Figura 1. Oocitos encontrados en hembras de Paracheirodon axelrodi bajo diferentes condiciones de $\mathrm{pH}$ (T1 y T2, ver texto). (A) Oocitos perinucleolares en diferentes etapas de desarrollo, (B) vista panorámica de corte sagital de una hembra en vitelogénesis, (C) oocito alvéolo cortical, (D) detalle de las membranas que rodean al oocito alvéolo cortical. (N) núcleo, (un) nucléolos, (Cb) citoplasma basófilo, (Op) grupo de oocitos perinucleolares, (oac) oocito alveolo cortical, (ov1) oocito vitelogénico en etapa inicial, (ov2) oocito vitelogénico en etapa tardía, (ac) alvéolos corticales, ( Vv) vesículas de vitelo, (Zp) zona pelúcida, (Mf) membrana folicular, (T) teca, (e) eritrocito. Barra: $50 \mu \mathrm{m}$. Tinción HyE.

Los tipos celulares observados en ambos tratamientos correspondieron con la siguiente descripción y se observan en las Figuras 1 y 2 :

Oocitos perinucleolares (Figura 1 - A): oocitos con citoplasma altamente basófilo, núcleo definido y presencia de numerosos nucléolos hacia la periferia de la membrana nuclear. Se observó una membrana folicular, como una capa de células alargadas con núcleo definido rodeando al oocito.

Oocito alveolo cortical (Figura 1 - C y D): oocito con citoplasma basófilo y vacuolizado, con presencia de alveolos corticales hacia la periferia de la membrana oocitaria. Se observaron pequeñas vesículas de vitelo eosinófilas y se diferenciaron tres membranas foliculares: la zona pelúcida, siendo eosinófila y sin presencia de núcleos celulares; la membrana folicular o 
granulosa, siendo esta una capa de células cilíndricas con núcleos redondeados y centrados; por último la teca como una capa simple de células alargadas y nucleadas.
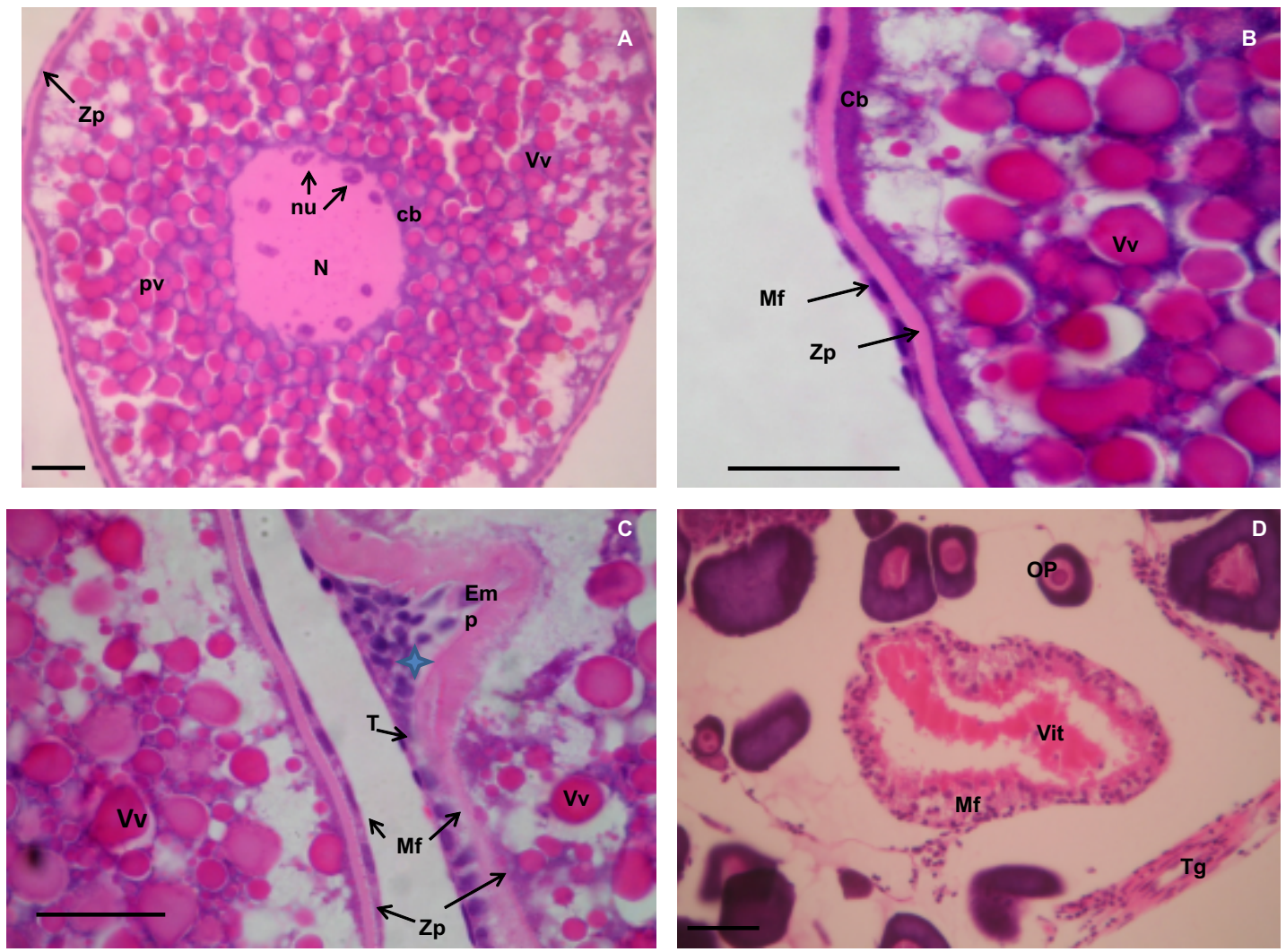

Figura 2. Oocitos encontrados en hembras de Paracheirodon axelrodi bajo diferentes condiciones de $\mathrm{pH}$ (A) oocito vitelogénico en etapa inicial, (B) detalle de las membranas presentes en el oocito vitelogénico en etapa inicial, (C) detalle de las membranas en el oocito vitelogénico en etapa tardía y desarrollo de la estructura micropilar, (D) oocito en desarrollo de atresia. (Zp) zona pelúcida, (Vv) vesículas de de vitelo, (N) núcleo, (nu) nucléolos, (cb) citoplasma basófilo (Mf) membrana folicular, (T) teca, (Emp) estructura micropilar, (estrella azul) células de la estructura micropilar, (OP) oocito perinucleolar, (Vit) vitelo, (Tg) tejido germinal. Barra: $50 \mu \mathrm{m}$. Tinción HyE.

Oocito vitelogénico en etapa inicial (Figura 2 - A y B): se caracterizó por la presencia del núcleo en posición central y con algunas zonas basófilas en el citoplasma. En comparación con el oocito alvéolo cortical, las vesículas de vitelo son más grandes. En este tipo de oocito se diferenció la zona pelúcida y la membrana folicular que se caracterizó por tener células con núcleos ovalados; la teca no se distinguió fácilmente.

Oocito vitelogénico en etapa tardía (Figura 1 - B y Figura 2 - C): se caracterizó por presentar oocitos de mayor tamaño, comparado con el oocito vitelogénico inicial. Se observó vacuolización completa del citoplasma sin zonas basófilas. Se diferenció la zona pelúcida y membrana folicular con células cuyos núcleos se aprecian más alargados. En algunos casos se presentó la formación de la estructura micropilar, como una invaginación de la zona pelúcida y membrana folicular.

Oocito en desarrollo de atresia (Figura 2 - D): se caracterizó por la proliferación de células de la membrana folicular invadiendo el oocito, perdiendo forma y organización de la membrana. 


\section{Análisis de proporciones oocitarias}

De acuerdo con los porcentajes para cada tipo de oocito en todos los meses y en cada una de las hembras adultas analizadas, se estableció que la fase de madurez correspondía a la de vitelogénesis o en maduración. Con la anterior descripción, las hembras presentaron oocitos de tipo celular vitelogénico en etapa tardía, que corresponden a la segunda mayor proporción oocitaria de las gónadas.

En la Tabla 1 se observan los datos correspodientes a las proporciones oocitarias econtradas en cada muestreo para cada tratamiento. De acuerdo con la prueba de t, sólo unos grupos oocitarios presentaron diferencias significativas entre T1 y 2 : los oocitos perinucleolares en el muestreo dos $(\mathrm{P}=0.007558)$, los oocitos alveolo cortical en el muestreo uno y cinco $(\mathrm{P}=$ 0.002632 y $\mathrm{P}=<2.2 \mathrm{e} 16$ respectivamente), oocitos vitelogénicos primarios en el muestreo cuatro $(P=0.002049)$ y los oocitos atrésicos en el muestreo $5\left(P=<2.2 e^{-16}\right)$. Sin embargo, la tendencia general indicó que las proporciones para cada tipo celular se mantuvieron similares en cada muestreo y entre los tratamientos.

Tabla 1. Proporción oocitaria en tejido gonadal de hembras de Paracheirodon axelrodi, sometidas a dos tratamientos de $\mathrm{pH}$ por un periodo de seis meses.

\begin{tabular}{|c|c|c|c|c|c|c|}
\hline \multirow[t]{2}{*}{ Tiempo } & \multirow[t]{2}{*}{ Tratamiento } & \multicolumn{5}{|c|}{ Proporción oocitaria(\%) } \\
\hline & & Op & Ac & Ov1 & Ov2 & At \\
\hline \multirow{2}{*}{ M1 } & $\mathrm{T} 1$ & $55 \pm 16^{a}$ & $1 \pm 2^{a}$ & $1 \pm 2^{a}$ & $43 \pm 15^{a}$ & $0 \pm 1^{a}$ \\
\hline & $\mathrm{T} 2$ & $51 \pm 18^{a}$ & $10 \pm 6^{b}$ & $3 \pm 4^{a}$ & $31 \pm 22^{a}$ & $5 \pm 7 \mathrm{a}$ \\
\hline \multirow{2}{*}{ M2 } & $\mathrm{T} 1$ & $61 \pm 7^{a}$ & $5 \pm 4^{a}$ & $1 \pm 1^{a}$ & $31 \pm 6^{a}$ & $2 \pm 1^{a}$ \\
\hline & $\mathrm{T} 2$ & $46 \pm 6^{b}$ & $10 \pm 4^{a}$ & $6 \pm 6^{a}$ & $35 \pm 7^{a}$ & $3 \pm 2^{a}$ \\
\hline \multirow{2}{*}{ M3 } & T1 & $56 \pm 8$ & 0 & 0 & $44 \pm 9$ & 0 \\
\hline & $\mathrm{T} 2$ & $50 \pm 18^{a}$ & $1 \pm 2^{a}$ & $3 \pm 3^{a}$ & $44 \pm 21^{a}$ & $1 \pm 1^{a}$ \\
\hline \multirow{2}{*}{ M4 } & T1 & $65 \pm 8$ & 0 & 0 & $34 \pm 8$ & $0 \pm 1$ \\
\hline & $\mathrm{T} 2$ & $69 \pm 6^{a}$ & 0 & $4 \pm 3 b$ & $25 \pm 8^{a}$ & $1 \pm 1^{a}$ \\
\hline \multirow{2}{*}{ M5 } & T1 & $57 \pm 4^{a}$ & $0^{a}$ & $0^{a}$ & $42 \pm 5^{a}$ & $1 \pm 1^{a}$ \\
\hline & $\mathrm{T} 2$ & $51 \pm 2^{a}$ & $3 \pm 0 b$ & $2 \pm 3^{a}$ & $40 \pm 0^{a}$ & $3 \pm 0 b$ \\
\hline \multirow{2}{*}{ M6 } & $\mathrm{T} 1$ & $55 \pm 6^{a}$ & 0 & $0^{a}$ & $43 \pm 6^{a}$ & $1 \pm 1^{a}$ \\
\hline & $\mathrm{T} 2$ & $61 \pm 16^{a}$ & 0 & $1 \pm 2^{a}$ & $37 \pm 14^{a}$ & $1 \pm 1^{a}$ \\
\hline
\end{tabular}

${ }^{a, b}$ Letras diferentes indican diferencias significativas $(\mathrm{P}<0.05)$. T1: $\left.\mathrm{pH} 5.5-5.8\right)$, T2: $\left.\mathrm{pH} 7.0-7.5\right)$. (Op) oocito perinucleolar, (Ac) oocito alveolo cortical, (Ov1) oocito vitelogénico en etapa inicial, (Ov2) oocito vitelogénico en etapa tardía, (At) oocitos en desarrollo de atresia. Los datos corresponden a la media \pm desviación estándar. 
De igual forma, el análisis estadístico de ANOVA indicó un valor de P >0.05, explicando que a través del tiempo no se presentaron diferencias en los tipos celulares, excepto para el tipo alveolo cortical de T1 $(P=0.0438)$ y de T2 $(P=0.000361)$.

En la Figura 3 A, B y C, se observan las gráficas correspondientes a las variables de crecimiento: longitud total, longitud estándar y peso, registradas a lo largo de 180 días de experimentación; de acuerdo al análisis estádistico no se presentaron diferencias significativas entre T1 y T2.
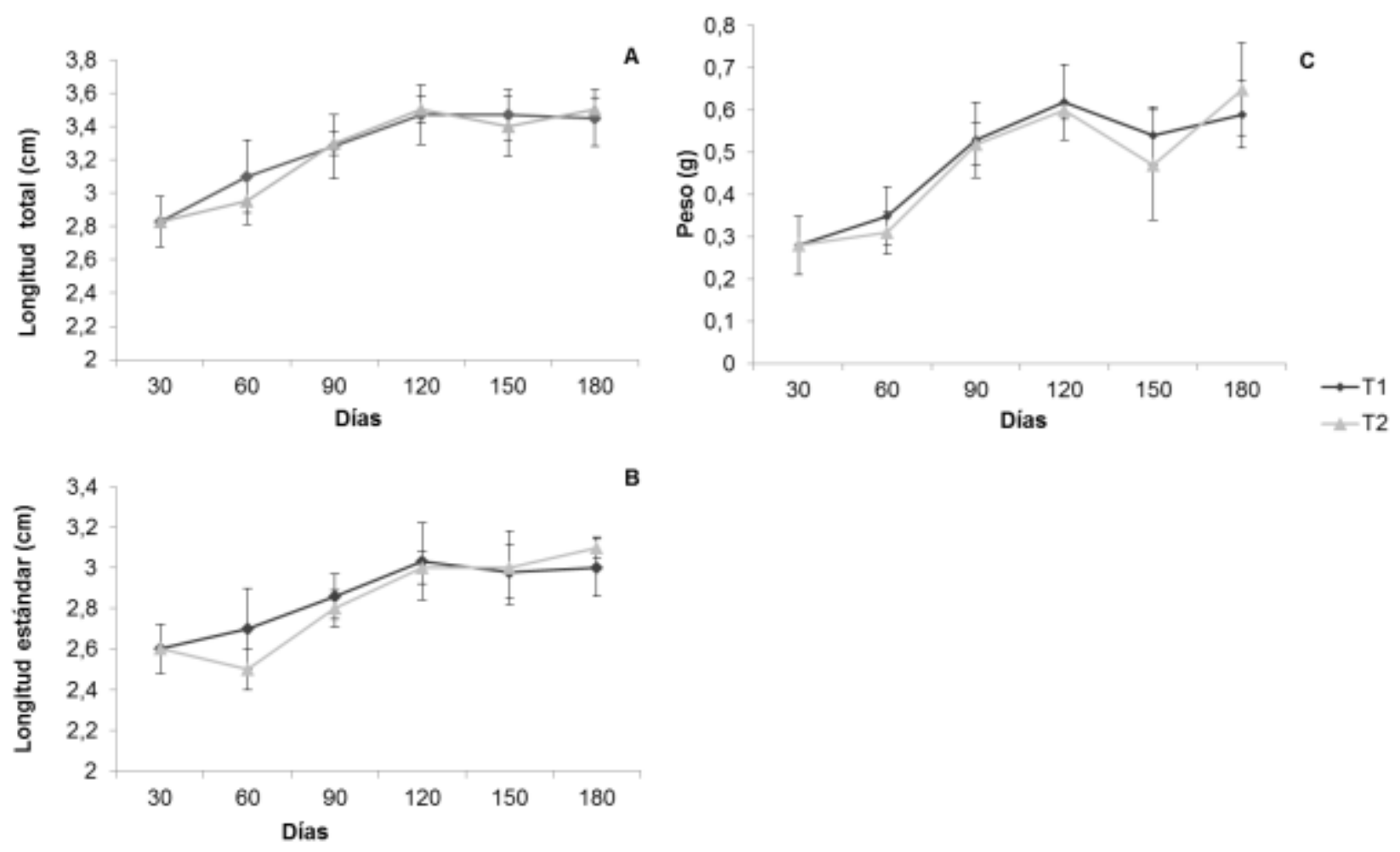

Figura 3. Variables de crecimiento registradas en hembras de Paracheirodon axelrodi durante 180 días de experimentación, bajo diferentes condiciones de pH (T1 y T2, ver texto). (A) longitud total, (B) longitud estándar, (C) peso. Los datos representan la media \pm deviación estándar.

Por otro lado, al comparar los valores promedio \pm desviación estándar de las variables de crecimiento al inicio y final del estudio, se observó que hubo un aumento en las variables. Ya que se inició con individuos de $2.8 \pm 0.15 \mathrm{~cm} \mathrm{LT}, 2.6 \pm 0.12 \mathrm{~cm}$ LS y $0.28 \pm 0.07$ g de peso y se finalizó con individuos de $3.45 \pm 0.17 \mathrm{~cm} \mathrm{LT}, 3.0 \pm 0.14 \mathrm{~cm} \mathrm{LS} ; 0.59 \pm 0.08 \mathrm{~g}$ en el tratamiento 1 y $3.5 \pm 0.07 \mathrm{~cm} \mathrm{LT}, 3.1 \pm 0.01 \mathrm{LS}, 0.65 \pm 0.11 \mathrm{~g}$ para el tratamiento 2.

\section{Discusión}

La descripción histológica de los tipos celulares pertenecientes a la línea oocitaria coincide con algunas de las características mencionadas por Brito y Bazzoli (2009), para la oogénesis de $P$. axelrodi donde se reportan cuatro estados de desarrollo oocitario: oocito perinucleolar inicial, perinucleolar avanzado, previtelogénico y vitelogénico. De acuerdo a las descripciones histológicas realizadas en el presente estudio, los oocitos alveolo corticales comparten características similares descritas para el oocito en estado previtelogénico; 
mientras que los oocitos vitelogénicos se caracterizaron por presentar dos etapas de desarrollo, una inicial y una tardía, no descritas en Brito y Bazzoli (2009). La descripción histológica de estas dos etapas es similar a la propuesta por Ocampo (2008) para la especie Sardinops sagax, en la que se describe una vitelogénesis inicial, caracterizada por tinciones HyE en los que se observa citoplasma basófilo con la presencia de vesículas de vitelo, y una vitelogénesis avanzada, donde además del incremento en el tamaño celular, el citoplasma se observa completamente eosinófilo; indicando que hay un cese de actividad para la formación de organelos y se da paso a la completa acumulación de vitelo (Lubzens, et al., 2010).

En el caso de la estructura micropilar, varios autores relacionan su formación con la fase de maduración del oocito, como una estructura que servirá para la entrada del espermatozoide al momento de la fertilización (Lubzens et al., 2010). Las características histológicas del micrópilo son similares a las que describen Brito y Bazzoli (2009). Sin embargo, no se clasificó al oocito de $P$. axelrodi como maduro, ya que en otras especies como $S$. sagax (Ocampo, 2008), además del micrópilo, se tiene en cuenta la migración del núcleo o vesicula germinal hacia el polo animal; característica que no se observó.

Por otro lado, la presencia de oocitos en desarrollo de atresia puede obedecer a un proceso común que ocurre en cualquier tipo celular oocitario, como una forma de aprovechamiento energético (Miranda et al., 1999). Este proceso puede incrementar su incidencia cuando los organismos se someten a algún tipo de estrés, como mala alimentación y/o condiciones fisicoquímicas del agua inapropiadas para un desove (Brito y Bazzoli, 2009). En este trabajo no hubo variaciones en las proporciones de oocitos atrésicos y éstas fueron comparativamente las más bajas con respecto de los otros tipos celulares.
Lo anterior refleja que las condiciones fisicoquímicas del agua, el tipo y frecuencia de alimentación en ambos tratamientos, permitieron que las hembras permanecieran durante seis meses en una misma fase de madurez gonadal (vitelogénesis). Este tiempo se considera superior a los periodos de maduración (meses junio y septiembre), que se han reportado en hembras de $P$. axelrodi capturadas en la región de Puerto Gaitán (Meta - Colombia); meses en los cuales se presentan la mayor proporción de oocitos en vitelogénesis (Velásquez, et al., 2008).

Teniendo en cuenta los tipos celulares encontrados en el tejido gonadal, se observó que ambos tratamientos mantuvieron las mismas proporciones oocitarias durante los meses de muestreo. Lo anterior indicó que los rangos de $\mathrm{pH}$ utilizados no tuvieron efecto sobre la proporción oocitaria; sin embargo, las condiciones a las que estuvieron expuestos los individuos, permitieron que se mantuvieran hembras en cautiverio con un estado de madurez avanzado por aproximadamente seis meses. Esta característica podría permitir manipular otros parámetros fisicoquímicos que puedan incidir en la maduración final (Burton, 1997).

Estudios reportados por Chapman et al. (1998) y Kucharczyk et al. (2010) en Paracheirodon innesi, una especie cercana filogenéticamente a $P$. axelrodi, demuestran la influencia de los factores ambientales en la reproducción. En esta especie, se crían y mantienen los peces reproductores en valores de $\mathrm{pH}$ de 6.9 - 7.4, pero el desove se hace en $\mathrm{pH}$ de $5.5-6.5$; adicional a esto se combina con descenso en el valor de la conductividad eléctrica (CE) y un aumento en la temperatura del agua. Por otro lado Ancistrus triradiatus, una especie tropical que comparte hábitats similares a $P$. axelrodi, madura gonadalmente en una CE de 100 $\mu \mathrm{S} / \mathrm{cm}$, pero el desove se alcanza al realizar un cambio súbito de los ejemplares a un medio con $80 \mu \mathrm{S} / \mathrm{cm}$ (Collazos y Arias, 2009). Por lo anterior, es posible que una combinación y 
cambios en variables como CE, pH y temperatura desencadenen el proceso de maduración final y la reproducción de $\mathrm{P}$. axelrodi, como se observa en los trabajos realizados por Burton (1997) y Anjos y Anjos (2006).

Otro aspecto relacionado con las condiciones mantenidas en el experimento, es proporcionado por los parámetros de crecimiento, pues aunque no se presentaron diferencias significativas entre los tratamientos, los peces crecieron considerablemente desde el inicio hasta el final del estudio, obteniendo que hembras adultas de $P$. axelrodi incrementarán su longitud y duplicarán su peso.

De acuerdo a lo anterior, las hembras analizadas en este estudio alcanzaron un rango de peso y longitud similar a los datos que se reportan de individuos en cautiverio, en donde hembras de $P$. axelrodi con tamaños de $3.1 \pm 0.5 \mathrm{~cm}$ de longitud total y peso $0.50 \pm$ 0.5 g se consideran maduras y se utilizan para desoves artificiales (Anjos y Anjos, 2006). Así mismo, en ambiente natural hembras con tamaños de $3.2 \mathrm{~cm}$ de longitud total alcanzan su madurez sexual en un periodo igual o superior a los 12 meses de edad (Geisler y Annibal, 1987). Sin embargo, al no conocer la fecha de eclosión de los peces utilizados en nuestro experimento, es difícil asegurar una edad en la que comenzó el proceso de maduración gonadal.

Con los resultados obtenidos en esta investigación, se puede concluir que al exponer hembras adultas de $P$. axelrodi a rangos de $\mathrm{pH}$ de 5.5 - 5.8 y $7.0-7.5$, no presentan un efecto en la proporción oocitaria. Sin embargo, las condiciones restantes permitieron mantener hembras en un estado de madurez avanzado por aproximadamente seis meses. Si se logra determinar con mayor precisión cuál o cuáles son los factores implicados en la maduración final y posterior desove, este trabajo ayudaría a piscicultores de peces ornamentales, pues les permitiría tener hembras relativamente maduras por periodos prolongados; generando varios lotes de reproductores, que podrían ser usados en varios desoves para obtener alevinos de manera escalonada. Por consiguiente, si se establecen estos protocolos se podría reproducir los peces en los momentos de mayor precipitación, ya que son las épocas en las que se requiere más esfuerzo de pesca y su captura es baja (Ramírez et al., 2001).

\section{Agradecimientos}

Los autores agradecen a la Universidad Militar Nueva Granada por la financiación del proyecto CIAS 1178. A Edna Rocio Riaño Castillo y Oswaldo Tovar Bohórquez por su colaboración durante la realización del estudio.

\section{Referencias}

Anjos HDB, Anjos CR. Biologia reprodutiva e desenvolvimento embrionário e larval do cardinal tetra, Paracheirodon axelrodi Schultz, 1956 (Characiformes: Characidae), em laboratório. B. Inst. Pesca, São Paulo. 2006; 32(2): 151-160.

Arias CJA, Martínez CPS, Romo ELD. Experimentos de levante del cardenal Paracheirodon axelrodi. Memorias XIII Jorn Acuic-IALL. Universidad de los Llanos, Villavicencio. 2007. p 34-36.

AVMA - Guidelines on Euthanasia (Formerly Report of the AVMA Panel on Euthanasia). American Veterinary Medical Association. 2007.

Brito MFG, Bazzoli N. Oogenesis of the cardinal tetra Paracheirodon axelrodi Schultz (1956): a histological and histochemical study. Braz. J Morphol Sci. 2009; 26(1): 14-18. 
Burton S. The effect of environmental factors and hormone on ovulation rate and spawning succes in cardinal tetras (Paracheirodon axelrodi) (pisces: Characidae). Thesis Submitted in fulfillment of the requirements for the Degree of MASTER OF SCIENCE Rhodes university, Grahamstown South Africa. 1997.

Chapman F, Colle D, Rottmann R, Shireman J. Controlled spawning of the Neon Tetra. The progressive Fish-culturist. 1998; 69: 32-37.

Collazos-Lasso LF, Arias-Castellanos JA. Estimulación a la maduración final y desove de Ancistrus triradiatus. Orinoquia. 2009; 13(1): 14-19.

Cooke G, Chao N, Beheregaray L. Phylogeography of a flooded forest specialist fish from central Amazonia based on intron DNA: the cardinal tetra Paracheirodon axelrodi. Freshwater Biology. 2009; 54: 1216- 1232.

Geisler R, Annibal S. Ecology of the Cardinal Tetra, Paracheirodon axelrodi (Pisces, Characoidea), in the River Basin of the Rio Negro, Brazil, as well as breeding related factors. Trap. Fish Hohhyisf, 1987; 35 (2): 66-87. En: Burton S. The effect of environmental factors and hormone on ovulation rate and spawning succes in cardinal tetras (Paracheirodon axelrodi) (pisces: Characidae).Thesis Submitted in fulfillment of the requirements for the Degree of MASTER OF SCIENCE Rhodes university, Grahamstown South Africa 1997.

Gómez-Ramírez E, Obando MJ, Tovar MO, Caldas ML y Hurtado H. Estudio histológico del tracto digestivo del Neón Cardenal Paracheirodon axelrodi (Characidae). Int J Morphol. 2011; 29(3): 782-786.
Kucharczyk D, Targońska K, Żarski D, Krejszeff S, Kupren K, Łuczyński M y Szczerbowski A. The reproduction of Neon Tetra, Paracheirodon innesi (Myers, 1936), under controlled conditions. Pol J Natur Sc. 2010; 25(1): 81-92.

Lubzens E, Young G, Bobe J, Cerdà J. Oogenesis in teleost: How fish eggs are formed. General and Comparative Endocrinology. 2010; 165(3): 367-489.

Mancera-Rodríguez NJ, Álvarez-León R. Comercio de peces ornamentales en Colombia. Acta biol Colomb. 2008; 13(1): $23-52$.

Miranda AC, Bazzoli N, Rizzo E, Sato Y. 1999. Ovarian follicular atresia in two teleost species: a histological and ultrastructural study. Tissue \& Cell. 1999; 31(5): 480 - 488.

Obando-Bulla MJ, Gómez-Ramírez E, TovarBohórquez MO, Rincón L, Caldas-Martínez ML, Hurtado-Giraldo H. Estudio morfométrico y topológico del cerebro del pez Neón Cardenal, Paracheirodon axelrodi (Characiformes: Characidae). Actu Biol. 2013; 35(98):45-61.

Ocampo J. Factores endógenos asociados al crecimiento del oocito y desove en Sardinop ssagax (Jenyns, 1842) (Teleostei: Clupeidae). Trabajo de grado para obtener el título de Doctor en Ciencias Marinas. Centro interdisciplinario de ciencias Marinas del Instituto Politécnico Nacional (IPN). La Paz, baja California Sur- México. 2008.

Oliveira S, Souza R, Nunes E, Carvalho C, Menezes G, Marcon J, Roubach R, Akifumiono E, Affonso E. Tolerance to temperature, $\mathrm{pH}$, ammonia and nitrite in 
cardinal tetra, Paracheirodon axelrodi, an amazonian ornamental fish. Acta Amaz. 2008; 38(4):773-780.

Ramírez H, Ajiaco R, Carrillo L. La pesca de especies ornamentales en el área de influencia de Puerto Carreño. En: Ramírez H y Ajiaco R. La Pesca En La Baja Orinoquia Colombiana: Una Visión Integral. 2001 Colombia. v.1.p.123-137.

Ravaglia A, Maggese MC. Oogenesis in the swamp e el Synbranchus marmoratus (Bloch, 1795) (Teleostei; synbranchidae). Ovarian anatomy, stages of oocyte development and micropyle structure. Biocell. 2002; 26(3): 325-337.

Reading B, Sullivan C. Vitellogenesis in Fishes. En: Farrell A. Encyclopedia of fish physiology: from genome to environment. 2011. San Diego US. p. 635 - 646.

SIPA - Sistema de Información Pesca y Acuicultura. Boletín pesquero Colombiano año 2011. Editorial Corporación Colombia Internacional. Bogotá Colombia.

Sloman K. The diversity of fish reproduction. En: Farrell A. Encyclopedia of fish physiology: from genome to environment. 2011. San Diego US. p. 613-615.

Tovar MO, Obando MJ, Gómez E, Caldas ML, Hurtado H. Histología y morfometría del ojo del pez dulceacuícola Paracheirodon axelrodi (characiformes: characidae). Rev Biol Trop. 2009; 57(4): 1107-1118.

Tyler CR, Sumpter JP. Oocyte growth and development in teleost. Reviews in Fish Biology and Fisheries. 1996; 6(3): 287-318.

Ureña F, Mora J, Landines M, Sanabria A. Capítulo 3, Tetras. En: Landines M,
Sanabria A, Daza P (Editores). Producción de peces ornamentales en Colombia. Ministerio de Agricultura y desarrollo Rural Instituto INCODER. 2007. Bogotá D.C, Colombia. p 48- 49.

Velásquez C, Moreno P, Muñoz M, Gómez E, Tovar O, Obando M, Hurtado H. Ciclo gonadal de neón cardenal (Paracheirodon axlelrodi) capturado en Puerto Gaitán (Meta), Colombia. Memorias IV Congreso Colombiano de Acuicultura. Rev Col Cienc Pec. 2008; $21: 455-522$. 